\title{
Age-related changes in the distributions of depressive symptom items in the general population: Cross-sectional study using the exponential distribution model
}

Shinichiro Tomitaka, Yohei Kawasaki, Kazuki Ide, Hiroshi Yamada, Toshiaki A Furukawa, Yutaka Ono

Background: Previous research has reported inconsistent evidence of the trajectory of depressive symptoms across the adult lifespan. We investigated how the distributions of each item score change with age and determined whether the trajectory of depressive symptoms varied with the scoring methods of the questionnaire.

Methods: We analyzed data collected from 21,040 subjects who participated in the national survey in Japan. Depressive symptoms were assessed using the Center for Epidemiologic Studies Depression Scale (CES-D). The CES-D has 20 items, each of which is scored in four grades of "rarely," "some," "much," and "most of the time." We used the exponential distribution model which fits the distributions of 16 negative symptom items of CES-D, with the probabilities of "some," "much," "most," and "rarely" expressed as P, Pr, $\mathrm{Pr}^{2}$, and 1-Px $\left(\mathrm{r}^{2}+\mathrm{r}+1\right)$.

Results: The distributions of the responses to 16 negative symptom items followed the common exponential model across all age groups. The mean of the estimated parameter $r$ of 16 negative items showed a U-shape pattern, being high during 12-29 years, remaining low during 30-50 years, and then increasing again over 60 years. The trajectory of depressive symptom scores simulating the binary method was different from that of the empirical scores using Liker method.

Conclusions: Our findings show that the increase in the depressive symptoms score during older age is based on the increase of the parameter $r$. The differences in the scoring method may contribute to the different age-related patterns across the adult lifespan. 
1 Age-related changes in the distributions of depressive symptom items in the

2 general population: Cross-sectional study using the exponential distribution

3 model

4 Shinichiro Tomitaka ${ }^{1,2}$, Yohei Kawasaki², Kazuki Ide $^{2}$, Hiroshi Yamada ${ }^{2}$, Toshiaki Furukawa ${ }^{3}$,

5 Ono Yutaka ${ }^{4}$

6 1) Department of Mental Health, Panasonic Health Center, Tokyo, Japan

7 2) Department of Drug Evaluation and Informatics, Graduate School of Pharmaceutical Sciences,

8 University of Shizuoka, Shizuoka, Japan

9 3) Department of Health Promotion and Human Behavior, Department of Clinical Epidemiology,

10 Kyoto University Graduate School of Medicine/School of Public Health, Kyoto, Japan

11 4) Center for the Development of Cognitive Behavior Therapy Training, Tokyo, Japan

13 Corresponding author:

14 Shinichiro Tomitaka ${ }^{1,2}$

15 Landic building, Nishi-shinbashi 3-8-3, Minato-ku, Tokyo, 105-003, Japan

16 Email address: tomitaka.shinichiro@jp.panasonic.com 


\section{Abstract}

19 Background: Previous research has reported inconsistent evidence of the trajectory of

20 depressive symptoms across the adult lifespan. We investigated how the distributions of each

21 item score change with age and determined whether the trajectory of depressive symptoms

22 varied with the scoring methods of the questionnaire.

23 Methods: We analyzed data collected from 21,040 subjects who participated in the national

24 survey in Japan. Depressive symptoms were assessed using the Center for Epidemiologic Studies

25 Depression Scale (CES-D). The CES-D has 20 items, each of which is scored in four grades of

26 "rarely," "some," "much," and "most of the time." We used the exponential distribution model

27 which fits the distributions of 16 negative symptom items of CES-D, with the probabilities of

"some," "much," "most," and "rarely" expressed as $\mathrm{P}, \mathrm{Pr}, \mathrm{Pr}^{2}$, and 1-P× $\left(\mathrm{r}^{2}+\mathrm{r}+1\right)$.

29 Results: The distributions of the responses to 16 negative symptom items followed the common

exponential model across all age groups. The mean of the estimated parameter $r$ of 16 negative

items showed a U-shape pattern, being high during 12-29 years, remaining low during 30-50

years, and then increasing again over 60 years. The trajectory of depressive symptom scores

33 simulating the binary method was different from that of the empirical scores using Liker method.

34 Conclusions: Our findings show that the increase in the depressive symptoms score during older

35 age is based on the increase of the parameter $r$. The differences in the scoring method may 
36 contribute to the different age-related patterns across the adult lifespan. 


\section{Introduction}

Depression, a common mental disorder, is one of the leading causes of disability worldwide

40 (Moussavi et al., 2007). There has been much interest in understanding the distribution of

41 depressive symptoms in the general population, given their association with depression (Blazer

42 \& Kessler, 1994; Kroenke et al., 2009).

43 Cross-sectional surveys and longitudinal studies, majority of which used the Center for

44 Epidemiologic Studies Depression Scale (CES-D), have found that the trajectory of depressive

45 symptoms across the adult lifespan follows a U-shaped pattern, with symptoms being high

46 during young adulthood, decreasing during middle adulthood, and then increasing again over the

47 age of 70 (Kessler et al., 1992; Oh et al., 2013; Sutin et al., 2013; Tomitaka et al., 2015a).

48 Conversely, some studies, particularly cross-sectional surveys using the Revised Clinical

49 Interview Schedule (CIS-R), consistently found that depressive symptoms were high during

50 middle adulthood and decreased over the age of 55 (Adult psychiatric morbidity in England,

51 2007; The Scottish Health Survey Volume 1).

52 The reason for the differences observed in age-related manifestations of depressive symptom

53 between CES-D and CIS-R is unclear. It is unlikely that the true effect of aging on depressive

54 symptoms in the general population is inconsistent, indicating a possible influence of

55 methodological factors on the results of epidemiological studies. 
56 The discrepancy between epidemiological studies could be explained by selection bias.

57 However, this explanation conflicts with the fact that even the large sample surveys in the randomly selected general population consistently show differences between CES-D and CIS-R

59 (Adult psychiatric morbidity in England, 2007; Tomitaka et al., 2015a).

60 Another possible explanation is the difference in choice of items between CES-D and CIS-R.

61 The CES-D consists of 16 negative symptom items and 4 positive affect symptom items (good,

62 hopeful, happy, and enjoyed) that are not included in CIS-R (Radloff, 1997). Conversely, CIS-R

63 contains 14 negative symptom items (somatic symptom, fatigue, concentration, sleep, irritability,

64 worry about physical health, depression, depressive ideas, worry, anxiety, phobias, panic, compulsions, and obsessions), some of which (e.g., panic, compulsion, and obsession) are not included in CES-D (Lewis et al., 1992) However, even the common items (e.g., depression,

67 depressive ideas, sleep, anxiety, and fatigue) show differences in age-related patterns between CES-D and CIS-R (Adult psychiatric morbidity in England, 2007; The Scottish Health Survey Volume 1; Cooper et al., 2015). Therefore, it is difficult to attribute these differences between CES-D and CIS-R to the choice of items; it is necessary to consider why even the same items

71 often differ. one question which is scored using a four point Likert method (0-1-2-3) based on four possible 
74 answers: "rarely or none of the time (rarely)," "some or little of the time (some)," "occasionally

75 or a moderate amount of time (much)," and "most or all of the time (most)" (Radloff, 1997).

76 Conversely, each item of CIS-R has two mandatory questions to screen the specific symptom

77 (Lewis et al., 1992). If responses indicate the specific symptom, four questions are asked and scored using a binary method $(0-1)$. The four non-mandatory questions contribute a single point to the $0-4$ scale for each item (except depressive idea which has a $0-5$ scale). The severity of each symptom appears to weigh more heavily in the CES-D scale than in the CIS-R scale. These

81 differences in the scoring method may contribute to the different age-related patterns observed across the adult lifespan. In support of this speculation, other studies investigating binary method questionnaires (e.g., Bradburn Affect Balance Scale) also reported that negative affect symptom items were higher during middle adulthood than during old age (Stacey \& Gatz 1991; Charles et al., 2001). To elucidate the effects of different scoring methods on each item score, it is necessary to understand how the distributions of each item score change with age. Therefore, a method for representing the distribution is necessary in order to detect age-related changes.

Using a variety of multivariate statistical methods, e.g. factor analysis, principal components analysis and cluster analysis, a lot of population studies on depressive symptoms have been performed so far. A common feature of these multivariate statistical methods is that they are

91 focused on the interrelationship of each variable. Even though the interrelationship of each 
92 variable has been overwhelmingly investigated by many researchers, to the best of our

93 knowledge, little work has been done to elucidate the mathematical patterns of the distribution of

94 each depressive symptom. Thus, in the previous study, we investigated the mathematical patterns

95 of the individual distributions for each item of the CES-D.

96 Although the method of the previous study was simple (just a histogram), we found an

97 intriguing phenomenon. The distributions of the 16 negative items for CES-D commonly

98 exhibited exponential patterns between "some" and "most" response levels, while "rarely" was

99 not related to the exponential patterns between "some" and "most" (Tomitaka et al., 2015b).

100 Based on the findings, we proposed a mathematical model for the distribution of the 16 negative

101 symptom items, which were expressed by two parameters, $\mathrm{P}$ and $\mathrm{r}$, where $\mathrm{P}$ stands for the

102 probability of "some" and r stands for the equal ratio among "some," "much," and "most". The

103 probabilities of "some," "much," "most," and "rarely" are expressed as $\mathrm{P}, \operatorname{Pr}, \mathrm{Pr}^{2}$, and 1-P× $\left(\mathrm{r}^{2}+\right.$

$104 \mathrm{r}+1)$. We utilized this mathematical model to quantify the age-related changes in the

105 distribution of negative symptom items.

106 The present study used cross-sectional data from a large, nationally representative survey

107 conducted annually to evaluate the health status of a representative sample from the general

108 Japanese population (Ministry of Health, Labor and Welfare, Statistics and Information

109 Department, 2002). We analyzed more than 20,000 CES-D assessments performed in subjects 
110 between 12 and 89 years.

111 Here we investigated whether the age-related changes observed in the total CES-D score were

112 mainly due to the 16 negative symptoms. After confirming that the 16 negative symptoms were

113 responsible for the U-shaped pattern of the total CES-D score and the distribution of the 16

114 negative items followed the mathematical model across all age groups, we used the mathematical

115 model to examine how the distribution of these symptoms changed according to the age group.

116 Finally, we tested whether the conversion of each item score of CES-D from the standard Likert

117 method to the binary method could affect age-related changes in depressive symptoms across

118 adulthood.

\section{Methods}

121 This study used data from the Active Survey of Health and Welfare (ASHW) conducted by the Japanese Ministry of Health, Labor and Welfare in 2000 (Ministry of Health, Labor and Welfare, Statistics and Information Department, 2002). ASHW is an annual nationwide survey conducted by the Japanese Government to collect data necessary for policy making and health promotion in

125 compliance with the Statistics Law. The legal and ethical approval of ASWH was given by the Ministry of Health, Labor and Welfare, Japan. In 2000, ASHW examined depressive symptoms among a representative sample from the general Japanese population. To ensure that the sample 
128

129

130

131

132

133

134

136

was adequately representative, survey participants were selected from individuals aged $>12$ years

living in 300 communities in Japan. These communities were selected from 881,851 precincts identified in the 1995 Census using a stratified sampling design. Verbal informed consent was obtained from all the subjects. The data and methods used by the survey have been described in detail before (Ministry of Health, Labor and Welfare, Statistics and Information Department, 2002).

4 The questionnaire was returned by 32,729 respondents, and the response rate was not publicized by the Ministry of Health, Labor and Welfare and Health. However, the response rates for similar surveys conducted 3 and 4 years earlier were $87.1 \%$ and $89.6 \%$, respectively (Kaji et al., 2010). Therefore, we assumed that the response rate for the present survey was over $80 \%$.

The present study was secondary analysis of ASWH data. The Ministry of Health, Labor and Welfare examined our study and permitted us to analyze data from ASWH in compliance with the Statistics Law. The present study was approved by the ethics committee of Panasonic Health Center (approval number, 2014-1), and the procedures were carried out in accordance with the approved guidelines and regulations.

We excluded 1,394 respondents as we suspected the validity of their responses (i.e., those who answered "rarely" or "most" for all items, regardless of the nature of the item). A total of 9,588 respondents with missing information on one or more key study variables (i.e., depressive 
146 symptoms or age or sex) were also excluded from the sample. An additional 50 respondents over

14789 years of age were excluded as the number was too small to elucidate the pattern of

148 distribution. The final sample consisted of 20,990 respondents between 12 and 89 years.

149 Measures

150 Depressive symptoms were assessed using the Japanese version of the CES-D (Shima et al.,

151 1985). This 20-item scale assesses the frequency of a variety of depressive symptoms within the

152 previous week $[0=$ rarely or none of the time (less than 1 day), $1=$ some or little of the time $(1-2$

153 days), 2 = occasionally or a moderate amount of time (3-4 days), and $3=$ most or all of the time

154 (5-7days)], yielding a total score of 0-60 (Radloff, 1997). Higher scores indicate greater

155 psychological distress. The 20 items of the CES-D were grouped into the following four

156 subscales: depressive mood (items 3, 6, 9, 10, 14, 17, and 18), somatic and retarded activities

157 symptoms (items 1, 2, 5, 7, 11, 13, and 20), interpersonal relations (items 15 and 19), and

158 positive affect symptoms (items $4,8,12$, and 16). The positive affect symptom items were

reverse-scored. The 16 items of depressive mood, somatic and retarded activities symptoms, and

symptom items do not (Tomitaka et al., 2015b).

162 
164 The respondents were grouped into the following age groups: $12-19,20-29,30-39,40-49,50-$

$16559,60-69,70-79$, and 80-89 years. The final sample consisted of 20,990 respondents between

166 the ages of $12-89$ years (ages 12-19; $\mathrm{N}=2457$ [male; $\mathrm{n}=1269$ ], ages 20-29; $\mathrm{N}=3748$ [male;

$167 \mathrm{n}=1788$ ], ages 30-39; $\mathrm{N}=3761$ [male; $\mathrm{n}=1783$ ], ages 40-49; $\mathrm{N}=3629$ [male; $\mathrm{n}=1788$ ], ages 50-59;

$168 \mathrm{~N}=3569$ [male; $\mathrm{n}=1800$ ], ages 60-69; $\mathrm{N}=2253$ [male; $\mathrm{n}=1155$ ], ages 70-79; $\mathrm{N}=1161$ [male;

$169 \mathrm{n}=517$ ], ages 80-89; $\mathrm{N}=412$ [male; $\mathrm{n}=108$ ] ).

170 The first step was to compare the total CES-D score and each item score by age group, and to

171 confirm that the 16 negative symptom items followed a U-shaped pattern across adulthood,

172 resulting in the U-shaped pattern of the total CES-D score. This pattern of each negative item

173 was shown to exhibit the lowest score during 30-69 years (Tomitaka et al., 2015a).

174 Second, in order to confirm that the distribution of the 16 negative items follow the

175 mathematical model across all age groups, their histograms were evaluated for each age group.

176 The distributions between "some" and "most" were analyzed using a log-normal scale.

177 Third, the parameters $\mathrm{P}$ and $\mathrm{r}$ were estimated from empirical data to quantify the age-related

178 changes in the distribution of the 16 negative items. These parameters were assessed for each

179 item using the mathematical model as follows: $\mathrm{P}=$ probability of "some," $\mathrm{r}=$ (probability of

180 "much") / (probability of "some") + (probability of "most")/ (probability of "much") / 2. The

181 mean values of the parameters were calculated for each age group. 
182 Finally, to demonstrate the effect of the scoring methods on age-related changes in depressive

183 symptoms, the total CES-D score and 16 negative items scores were compared between the

184 standard Likert method and the binary method. We used JMP version 11 for Windows (SAS

185 Institute, Inc., Cary, NC, USA) to calculate the descriptive statistics and frequency distribution

186 curves.

187

\section{Results}

\section{Age-related changes of each item score and total CES-D score across adulthood}

The total scores of 20 items and 16 negative item scores exhibited a similar U-shaped pattern,

with symptoms being highest during 12-29 years, decreasing during 30-69 years, and then

pattern (Fig. 1). The similarity in the pattern of the total score of 20 items and the 16 negative

items score suggested that the U-shaped pattern of total CES-D score is mainly attributed to the age-related changes of the 16 negative symptom items. relations group exhibited U-shaped patterns (Table 1). All 16 negative symptom items were the 
200 years, indicating that the U-shaped pattern is mostly common to the 16 negative items. Only

201 three negative items belonging to the somatic and retarded activities symptoms subscale (i.e.,

202 "bothered," "sleep," and "talked") did not show the U-shaped pattern. "Bothered," "sleep," and

203 "talked" were the lowest during 12-19 years and highest during 80-89 years.

204 In contrast to the negative items, the relationship between age and positive affect symptom

205 items were mild and differed from each other. "Good" was the highest during 60-69 years,

206 "happy" was the highest during 70-79 years, and "hopeful" and "enjoyed" were the highest

207 during $80-89$ years.

208

209

\section{Confirming that the distribution of 16 negative items follow the mathematical model}

210 To confirm that they followed the mathematical model., the distributions of the 16 negative

211 items were evaluated among each group $(12-19,20-29,30-39,40-49,50-59,60-69,70-79$,

212 and 80-89 years ) (Fig. 2A, 2B, 2C, 2D, 2E, 2F, 2G and 2H).

The distribution of the 16 items showed a common pattern across all age groups (Figure 2).

214 The lines for the 16 items seemed to intersect at a single point between "rarely" and "some"

215 across all age groups. As previously reported, all the lines that follow this mathematical model

216 theoretically cross at a single point between "rarely" and "some" (Tomitaka et al, 2015b).

217 Conversely, the lines for "some" to "most" responses were regularly skewed towards "some." 

across all age groups, the graphs of the distributions appeared to change with age. This change in

220 the distribution with age was examined by focusing on "some" and "most" responses. As the red arrows indicate, the frequencies of "most" response for 16 items were decreasing during 12-39 years (Fig 2A, 2B and 2C), stable during 40-69 years (Fig 2D, 2E and 2F), and then increasing again during 70-89 years (Fig 2G and 2H). Conversely, as the blue arrows indicate, the frequencies of "some" response for 16 items were slightly increasing during 12-39 years (Fig 2A, 2B and 2C), decreasing during 40-69 years (Fig 2D, 2E and 2F) and were unclear during 70-89 years (Fig $2 \mathrm{G}$ and $2 \mathrm{H}$ ). It is necessary to quantify the age-related changes in the distributions of 16 negative items in order to evaluate the variations precisely. followed an exponential pattern with the same parameter $\mathrm{r}$ (Fig. 3). Upon examining by age groups, the slopes of the lines for 16 negative items with a log-normal scale were seen to change according to age $(12-19,20-29,30-39,40-49,50-59,60-69,70-79$, and 80-89 years ) (Fig. 3A, 3B, 3C, 3D, 3E, 3F, 3G and 3H). The slopes were more horizontal during 70-89 years (Fig 3G and $3 \mathrm{H}$ ) than during 30-69 years (Fig 3C, 3D, 3E and 3F). 
236

237

238

239

240

241

242

243

244

245

246

247

248

249

250

251

252

253

\section{Quantification of age-related changes in the distributions of 16 negative items}

The parameters $\mathrm{r}$ and $\mathrm{P}$ were estimated from empirical data to quantify the age-related changes in the distributions of 16 negative items. Figure 4 shows that the average of the estimated parameter $\mathrm{r}$ exhibited a U-shaped pattern, being high during 12-29 years, staying low during 30-59 years, and then increasing again during 60-89 years, similar to the U-shaped pattern of total CES-D score (Fig. 1). In contrast, the mean of the estimated parameter P was stable across all age groups as compared with the parameter $r$. The average of estimated parameter P slightly increased during 12-49 years, slightly decreased during 50-79 years, and then increased again during 80-89 years.

\section{Comparison of the Likert scoring and binary scoring}

Finally, to demonstrate the effects of scoring methods in adulthood, total CES-D score and 16 item CESD scores were compared between the standard Likert $(0,1,2,3)$ and binary methods $(0$ 1-1-1) (Fig. 5). The total CES-D score and16 item CESD scores in the binary methods were estimated from empirical data. We analyzed the respondents between 12-79 years as the older participants recruited by other studies were mainly under 80 years. In the standard Likert method, the total CES-D score and total 16-item score showed a U-shaped pattern (Fig. 5A). In contrast, in the binary method, the estimated total CES-D score and total 16-item score showed a 
254 downward trajectory with age (Fig. 5B). The total CES-D score and total 16-item score were

255 higher in the 70-79 year group than the 30-59 year group in the Likert method, but lower in the

$25670-79$ year group than the 30-59 year group in the binary method.

\section{Discussion}

259

The aim of the present study was to delineate the age-related changes in the distributions of

each item score, and to test whether the trajectory of depressive symptoms varies with the scoring method.

The main findings of this study are that (1) the U-shaped pattern of total CES-D score across adulthood is mainly attributed to the age-related changes of 16 negative symptoms, (2) the mathematical model fits the distributions of 16 negative items across the adult span, and (3) the estimated parameter $\mathrm{r}$ of 16 negative items showed a U-shaped pattern, being high during 12-29 years, staying low during 30-59 years, and then increasing again during 60-89 years.

267 The trajectory of depressive symptoms across adulthood could be explained by the 268 aforementioned findings. Theoretically, the expected value of each negative item score is $\mathrm{P} \times$ $269\left(3 r^{2}+2 r+1\right)$ with the Likert method $(0-1-2-3)$ and $P \times\left(r^{2}+r+1\right)$ with the binary method $(0-1-$

270 1-1). Because expected values include the square of $r$ and the changes of parameter $r$ across the 271 adult lifespan are more intense than that of parameter $\mathrm{P}$, the trajectory of depressive symptoms 
272 are mostly affected by parameter $\mathrm{r}$, resulting in the similar U-shaped patterns.

273 Our findings indicate that the increase of depressive symptoms for older age is based on the

274 increase in parameter $\mathrm{r}$, which corresponds to the increase of the probabilities of "much" and "most,"

275 suggesting that aging tends to induce more severe depressive symptoms. However, this

276 explanation conflicts with the fact that most epidemiological evidence suggests that major

277 depressive disorders decline with advancing age (Kessler et al., 2010). Generally, there is the

278 discrepancy between the fact that rates of clinical depression are highest in midlife, whereas

279 depressive symptom screening scale scores (using Likert method) are highest in older age

280 (Kessler 1992). It remains unclear whether people are more depressed with aging. One possible

281 explanation is that there may be an age-related change in self-rating for the severity of depressive

282 symptoms. It has been found that older individuals tend to have problems with retrieving

283 information that is highly specific because they are less effective at controlling their retrieval

284 process (Luo \& Craik 2009). Further studies are necessary to clarify the fact that depressive

285 symptom screening scale scores are highest in older age.

286 Although the findings of the present paper cannot explain the discrepancy between the rates of

287 clinical depression and depressive symptom screening scale scores, they could explain the

288 discrepancy between Likert method and binary method. Using the binary method, the total CES-

289 D and 16-item scores were lower in the 70-79 age group than in the 30-59 age group (Fig. 5B). 
290 This finding could be explained as follows. First, although the parameter $r$ increases during 60-

29189 years, the expected value using the binary method does not reflect the increase in parameter $r$

292 as much as the Likert method. Secondly, the parameter P decreases during 50-79 years, being

293 lowest during 70-79 years. Thus, the CES-D score using binary method exhibits lower scores

294 during 70-79 years than during 30-59 years (Fig. 5). Therefore, our studies support the

295 hypothesis that the trajectory of depressive symptoms across the adult lifespan varies with the

296 scoring method.

297 In addition to the scoring method, there is another methodological factor which may be

298 responsible for the inconsistent evidence of the trajectory of depressive symptoms. While the

299 total depressive symptom score follows a U-shaped pattern across adulthood, the age-related

300 changes in depressive symptoms seem relatively mild (Sutin et al., 2013). The estimated average

301 change per decade in CES-D total score is less than one point between the ages of 20 and 79

302 years, while the standard deviation of the CES-D scores in community surveys is between 5 and

30310 points (Kessler et al., 1992; Hek et al., 2013; Oh et al., 2013). The mean total CES-D scores

304 appear to stabilize between the ages of 30 and 69 years in particular. As Kessler et al. pointed out,

305 a number of studies have worked with samples having a truncated age range, a small number of

306 very old respondents (older than 75 years), or a measure of age that combines all respondents

307 over the age of 65 into a single group (Kessler et al., 1992). These truncated age ranges may 
308

309

310

311

312

cause the researcher to overlook the non-linear increase in depressive symptoms that occurs after

the age of 70 (Newmann, 1989; Kessler et al., 1992).

While 13 of the 16 negative symptom items exhibit U-shaped patterns across the adult lifespan,

the remaining three negative items belonging to the somatic and retarded activities symptoms subscale do not exhibit this pattern. "Bothered," "sleep," and "talked" are the lowest during 1219 years instead of 30-69 years, and the highest during 80-89 years. These results are congruent with previous reports that some items of the somatic and retarded activities symptoms subscale exhibit the lowest scores during young adulthood and the highest scores during older age (Berry et al., 1984; Hegeman et al., 2012).

In comparison with the16 negative symptom items, the age-related changes in four positive affect symptom items were mild and differed from each other in the present study. Most of the studies report that the trajectories of positive affect symptom items differ from those of negative affect symptoms, although the evidence for trajectories of positive affects symptoms has been somewhat mixed (Kunzmann, 2008). While the age-related changes in positive affect items were mild, the total scores of 4 positive item scores increased during 30-89 years. This finding is in line with Carstensen's report that emotional well-being mildly improves from early adulthood to old age (Carstensen et al, 2000).

Recently, because of their relative independence, positive affect and negative affect have been 
326

327

328

329

330

331

commonly recognized as two different phenomena that should be studied individually (Lucas et

al., 1995). A number of cross-cultural comparison studies have reported that the response patterns for the positive affects items vary according to ethnicity or nation (eg, skewed, plateaushaped, U-shaped and reverse U-shaped), whereas the response patterns for the 16 negative items were generally comparable (Iwata et al.1995, Iwata et al., 1998). Although the CES-D score is the composite score of the 20 -item scores, it could be appropriate to recognize the 16 negative item scores and the positive scores as different scores (Tomitaka et al., 2015b).

The present paper indicates that the mathematical model fits the distributions of 16 negative items across the adult span. The conditions that enable such a common distribution can be speculated upon. In general, self-rating depression scales are used as follows. First, each subject must determine whether a symptom is present. If the level of the symptom does not meet the threshold which excludes everyday mild symptoms, it is regarded as "rarely (less than 1 day)." Next, if a depressive symptom that meets the threshold is present, the duration of the symptom is quantified and divided into "some (1-2 days)," "much (3-4 days)," and "most (5-7 days)." This two-step process increases the possibility that "rarely" will cover the range that does not meet the threshold, while each of "some," "much," and "most" will cover the almost fixed ratio of the range that satisfies the threshold. If what we call the latent trait of depressive symptoms could be exponential distribution which has the memoryless property, the 16 negative symptom items for 
344 CES-D would commonly exhibit the mathematical distribution that was observed in the present

345 study. In general, exponential distribution is observed where individual variability and total

346 stability are organized together, such as the Boltzmann-Gibbs law and income distribution

347 (Dragulescu \& Yakovenko, 2000). With respect to individual variability and total stability, the

348 conditions that enable exponential distribution could be present in the distributions of the 16

349 negative items. Further consideration regarding this speculation is needed.

350 There are some methodological advantages in the present investigation. First, the sample was

351 a representative of the general Japanese population, which reduced selection bias. The large

352 sample size $(\mathrm{N}=21,040)$ enabled us to elucidate the patterns of the distributions of depressive

353 symptom items.

354 Second, the mathematical model helped understand the age-related changes in the distribution

355 of depressive symptoms. Because these distributions are completely different from normal

356 distribution, the parameters of normal distribution (e.g., average and standard deviation) were not

357 sufficient to express the distributions themselves. After assessing the fit of the mathematical

358 model to the distributions of depressive symptoms, we utilized it to quantify the age-related

359 changes in the distributions of depressive symptoms. To the best of our knowledge,

360 mathematical modeling is still not well developed in the field of psychiatry. While depressive

361 symptoms of individuals are difficult to predict, the entire population may follow a certain 
362 mathematical pattern (Tomitaka et al., 2015a).

363 This study has some limitations. First, a standard psychiatric diagnosis with structured

364 interview was not performed for the subjects in this study. Therefore, the study did not encompass a psychiatric diagnosis of depressive symptoms. Second, although we evaluated the fit of the mathematical model to the distributions of depressive symptoms, analysis based on other mathematical models was not performed in this study. In general, the most important part of model evaluation is testing whether the model fits empirical data better than other models.

However, to the best of our knowledge, no other mathematical models for the distributions of depressive symptoms have been reported so far. Despite these limitations, the present study provides important information regarding the age-related changes in depressive symptoms.

\section{Conclusion}

Our results indicate that the scoring method of depressive symptoms is important in evaluating the age-related changes in depressive symptoms. The proposed mathematical model fits the symptoms in the general population follow a mathematical pattern as a whole.

\section{Acknowledgements}


380 The authors would like to thank the Active Survey of Health and Welfare project for providing 381 the data for this study, Dr. Shinji Sakamoto for helpful advices, and Enago (www.enago.jp) for 382 the English language review. 
384

385

386

387

388

389

390

391

392

393

\section{References}

Adult psychiatric morbidity in England, 2007, Results of a household survey - The NHS

Information Centre for health and social care.

(http://www.hscic.gov.uk/catalogue/PUB02931/adul-psyc-morb-res-hou-sur-eng-2007-rep.pdf). Accessed July 7, 2015.

Berry JM, Storandt M, Coyne A (1984). Age and sex differences in somatic complaints associated with depression. Journal of Gerontology 39, 465-467.

Blazer DG, Kessler RC (1994). The prevalence and distribution of major depression in a national community sample: the National Comorbidity Survey. The American Journal of Psychiatry 151, 979-986.

Carstensen LL, Pasupathi M, Mayr U, Nesselroade JR. (2000). Emotional experience in everyday life across the adult life span. Journal of Personality and Social Psychology 79, 644-55.

Charles ST, Reynolds CA, Gatz M (2001). Age-related differences and change in positive and negative affect over 23 years. Journal of Personality and Social Psychology 80, 136-151.

\section{Cooper C, Rantell K, Blanchard M, McManus S, Dennis M, Brugha T, Jenkins R, Meltzer}

H, Bebbington P (2015). Why are suicidal thoughts less prevalent in older age groups? Age differences in the correlates of suicidal thoughts in the English Adult Psychiatric Morbidity Survey 2007. Journal of Affective Disorders 177, 42-48.

Dragulescu A, Yakovenko VM (2000). Statistical mechanics of money. The European Physical Journal B-Condensed Matter and Complex Systems 17, 723-729. 
404

405

406

407

408

409

410

411

412

413

414

415

416

417

418

419

420

421

422

423

424

425

Hegeman JM, Kok RM, Van der Mast RC, Giltay EJ (2012). Phenomenology of depression

in older compared with younger adults: meta-analysis. The British Journal of Psychiatry: the Journal of Mental Science 200, 275-281.

Hek K, Demirkan A, Lahti J, Terracciano A, Teumer A, Cornelis MC, Amin N, Bakshis E, Baumert J, Ding J, Liu Y, Marciante K, Meirelles O, Nalls MA, Sun YV, Vogelzangs N, Yu L, Bandinelli S, Benjamin EJ, Bennett DA, Boomsma D, Cannas A, Coker LH, de Geus E, De Jager PL, Diez-Roux AV, Purcell S, Hu FB, Rimm EB, Hunter DJ, Jensen MK, Curhan G, Rice K, Penman AD, Rotter JI, Sotoodehnia N, Emeny R, Eriksson JG, Evans DA, Ferrucci L, Fornage M, Gudnason V, Hofman A, Illig T, Kardia S, Kelly-Hayes M, Koenen K, Kraft P, Kuningas M, Massaro JM, Melzer D, Mulas A, Mulder CL, Murray A, Oostra BA, Palotie A, Penninx B, Petersmann A, Pilling LC, Psaty B, Rawal R, Reiman EM, Schulz A, Shulman JM, Singleton AB, Smith AV, Sutin AR, Uitterlinden AG, Völzke H, Widen E, Yaffe K, Zonderman AB, Cucca F, Harris T, Ladwig KH, Llewellyn DJ, Räikkönen K, Tanaka T, van Duijn CM, Grabe HJ, Launer LJ, Lunetta KL, Mosley TH Jr, Newman AB, Tiemeier H, Murabito J (2013). A genome-wide association study of depressive symptoms. Biological Psychiatry 73, 667-678.

Iwata N, Roberts CR, Kawakami N (1995). Japan-U.S. comparison of responses to depression scale items among adult workers. Psychiatry Research 58, 237-245.

Iwata N, Umesue M, Egashira K, Hiro H, Mizoue T, Mishima N, Nagata S (1998). Can positive affect items be used to assess depressive disorders in the Japanese population? Psychological Medicine 28, 153-158.

Kaji T, Mishima K, Kitamura S, Enomoto M, Nagase Y, Li L, Kaneita Y, Ohida T, 
426 Nishikawa T, Uchiyama M (2010). Relationship between late-life depression and life stressors:

427 large-scale cross-sectional study of a representative sample of the Japanese general population.

428 Psychiatry and Clinical Neurosciences 64, 426-434.

429 Kessler RC, Birnbaum H, Bromet E, Hwang I, Sampson N, Shahly V (2010). Age

430 differences in major depression: results from the National Comorbidity Survey Replication

431 (NCS-R). Psychological Medicine 40, 225-237.

432 Kessler RC, Foster C, Webster PS, House JS (1992). The relationship between age and

433 depressive symptoms in two national surveys. Psychology and Aging 7, 119-126.

434 Kroenke K, Strine TW, Spitzer RL, Williams JB, Berry JT, Mokdad AH (2009). The PHQ-

4358 as a measure of current depression in the general population. Journal of Affective Disorders

436 114, 163-173.

437 Kunzmann U (2008). Differential age trajectories of positive and negative affect: Further

438 evidence from the Berlin Aging Study. The Journals of Gerontology. Series B, Psychological

439 Sciences and Social Sciences 63, P261-P270.

440 Lewis G, Pelosi AJ, Araya R, Dunn G (1992). Measuring psychiatric disorder in the

441 community: a standardized assessment for use by lay interviewers. Psychological Medicine 22,

$442 \quad 465-486$.

Lucas RE, Diener E, Suh E (1996). Discriminant validity of well-being measures. Journal of

444 Personality and Social Psychology 71:616-28.

445 Luo L, Craik FI (2009). Age differences in recollection: Specificity effects at retrieval. Journal 
446 of Memory and Language 60, 421-436.

447 Ministry of Health, Labor and Welfare, Statistics and Information Department (2002)

448 Active survey of Health and Welfare Health_(in Japanese). Tokyo: Labor and Welfare Statistics

449 Association.

450 Moussavi S, Chatterji S, Verdes E, Tandon A, Patel V, Ustun B (2007). Depression, chronic

451 diseases, and decrements in health: results from the World Health Surveys. Lancet 370, 851-858.

452 Newmann JP (1989). Aging and depression. Psychology and Aging 4, 150-165.

453

454

455

456

457

458

459

Oh DH, Kim SA, Lee HY, Seo JY, Choi BY, Nam JH (2013). Prevalence and correlates of depressive symptoms in Korean adults: results of a 2009 Korean community health survey. Journal of Korean Medical Science 28, 128-135.

Radloff LS (1977). The CES-D scale: a self-report depression scale for research in the general population. Applied Psychological Measurement 1, 385-401.

Shima S, Shikano T, Kitamura T, Asai M (1985). A new self-rating depression scale (in Japanese). Psychiatry 27, 717-723.

Stacey CA, Gatz M (1991). Cross-sectional age differences and longitudinal change on the Bradburn Affect Balance Scale. Journal of Gerontology 46, P76-P78.

Sutin AR Terracciano A, Milaneschi Y, An Y, Ferrucci L, Zonderman AB (2013). The trajectory of depressive symptoms across the adult life span. JAMA Psychiatry 70, 803-811.

The Scottish Health Survey Volume 1: Main Report - A National Statistics Publication for Scotland. (http://www.gov.scot/resource/doc/358842/0121284.pdf) Accessed July 7, 2015. 
466 Tomitaka S, Kawasaki Y, Furukawa T (2015a). Right Tail of the Distribution of Depressive

467 Symptoms Is Stable and Follows an Exponential Curve during Middle Adulthood. Public

468 Library of Science One 10, e0114624.

469 Tomitaka S, Kawasaki Y, Furukawa T.(2015b) A distribution model of the responses to each

470 depressive symptoms item in a general population. Cross-sectional study. BMJ Open 5, e008599. 
1

The relationship between age and the total scores of 20 items, 16 negative items score, and 4 positive items score.

The total scores of 20 items (blue line) and 16 negative item scores (red line) exhibited a similar U-shaped pattern, whereas the 4 positive items (yellow line) showed a plateau-shaped pattern. 


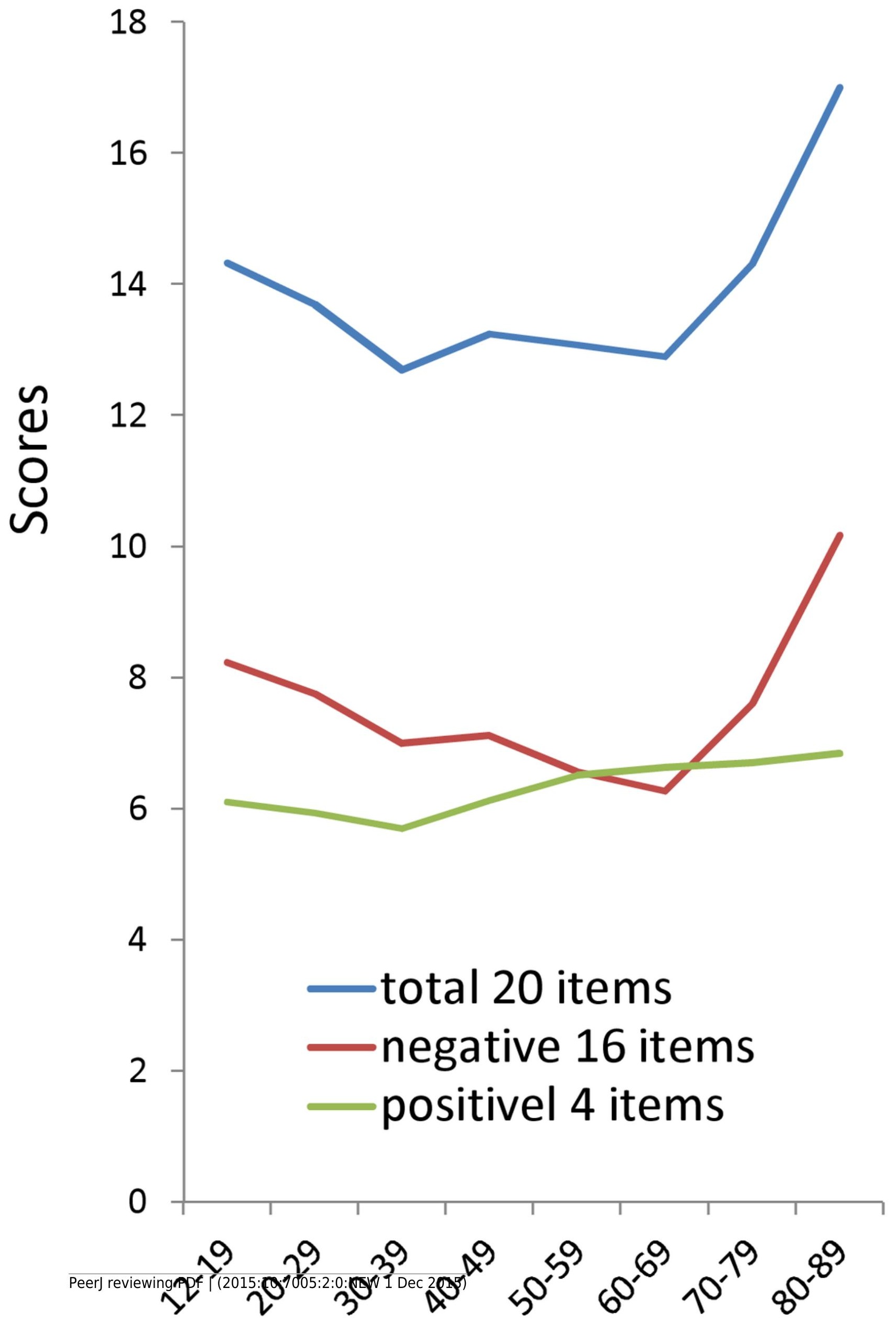


2

The distributions of 16 negative symptoms among each group

The distributions of 16 negative symptoms among each group, A) 12-19, B) 20-29, C) 30-39, D) 40-49, E) 50-59, F) 60-69, G) 70-79, and H) 80-89 years. Red arrows indicate the probability of "most" and blue arrows indicate the probability of "some." Although the distributions for each of the 16 items followed a common mathematical pattern across all age groups, the graphs of the distributions appeared to change with age.

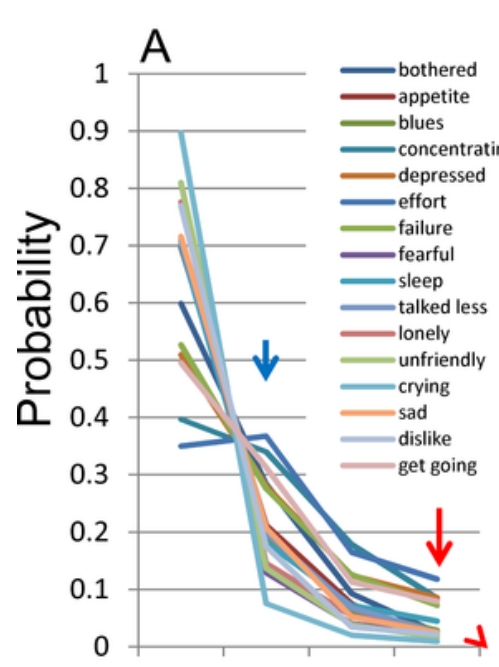

rarely some much most

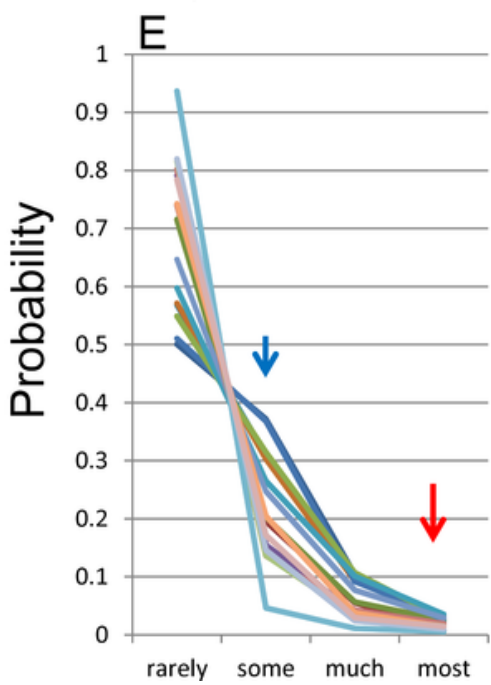

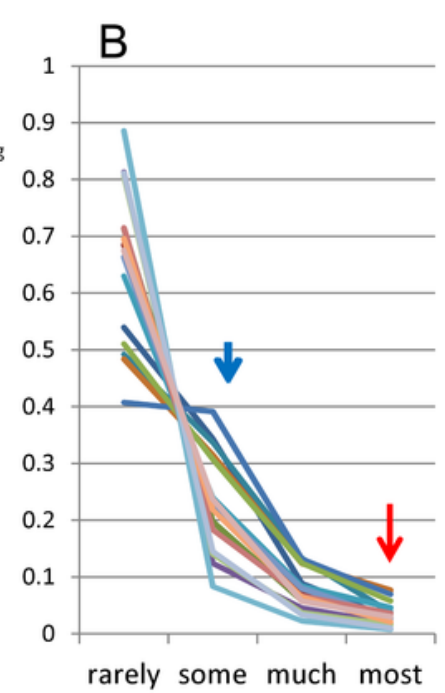
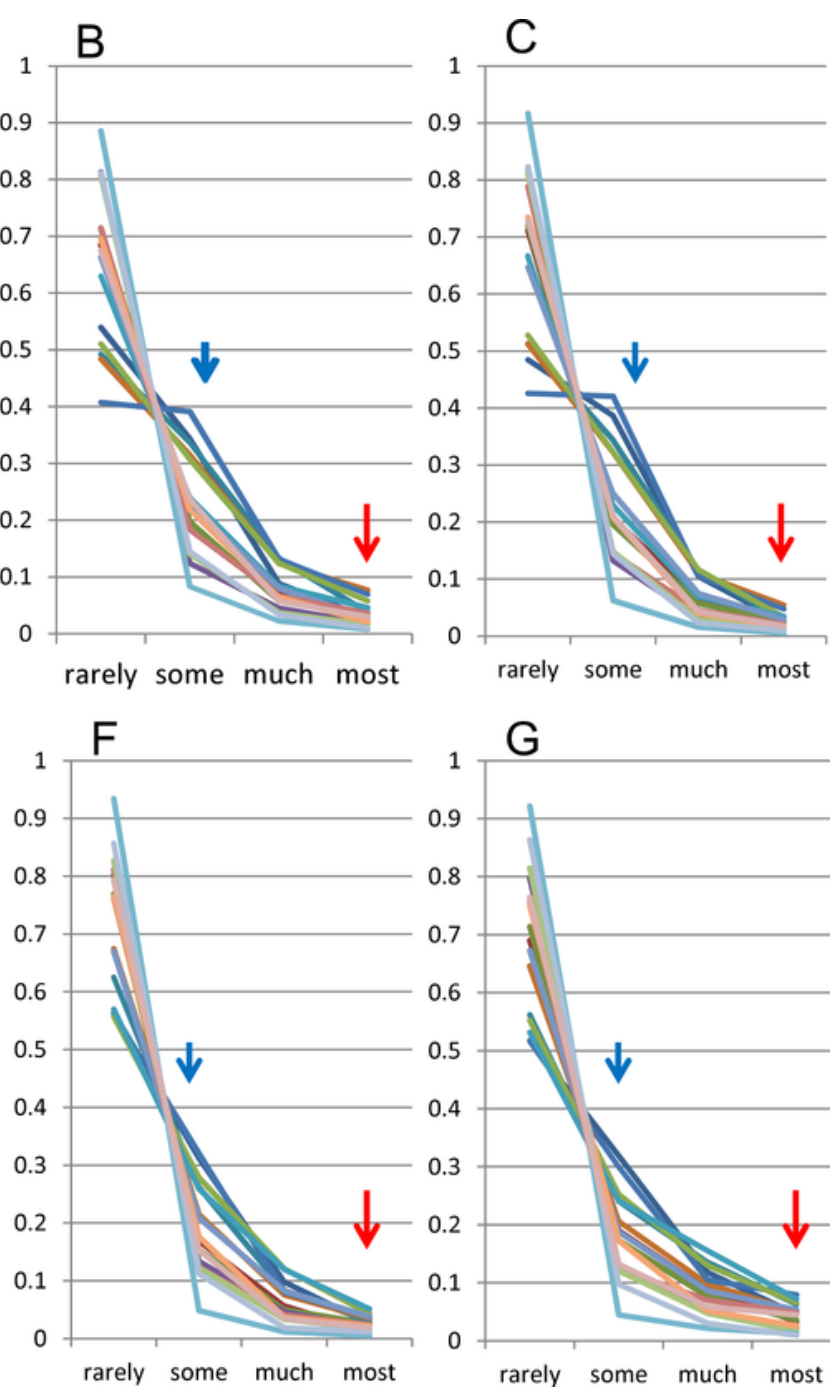
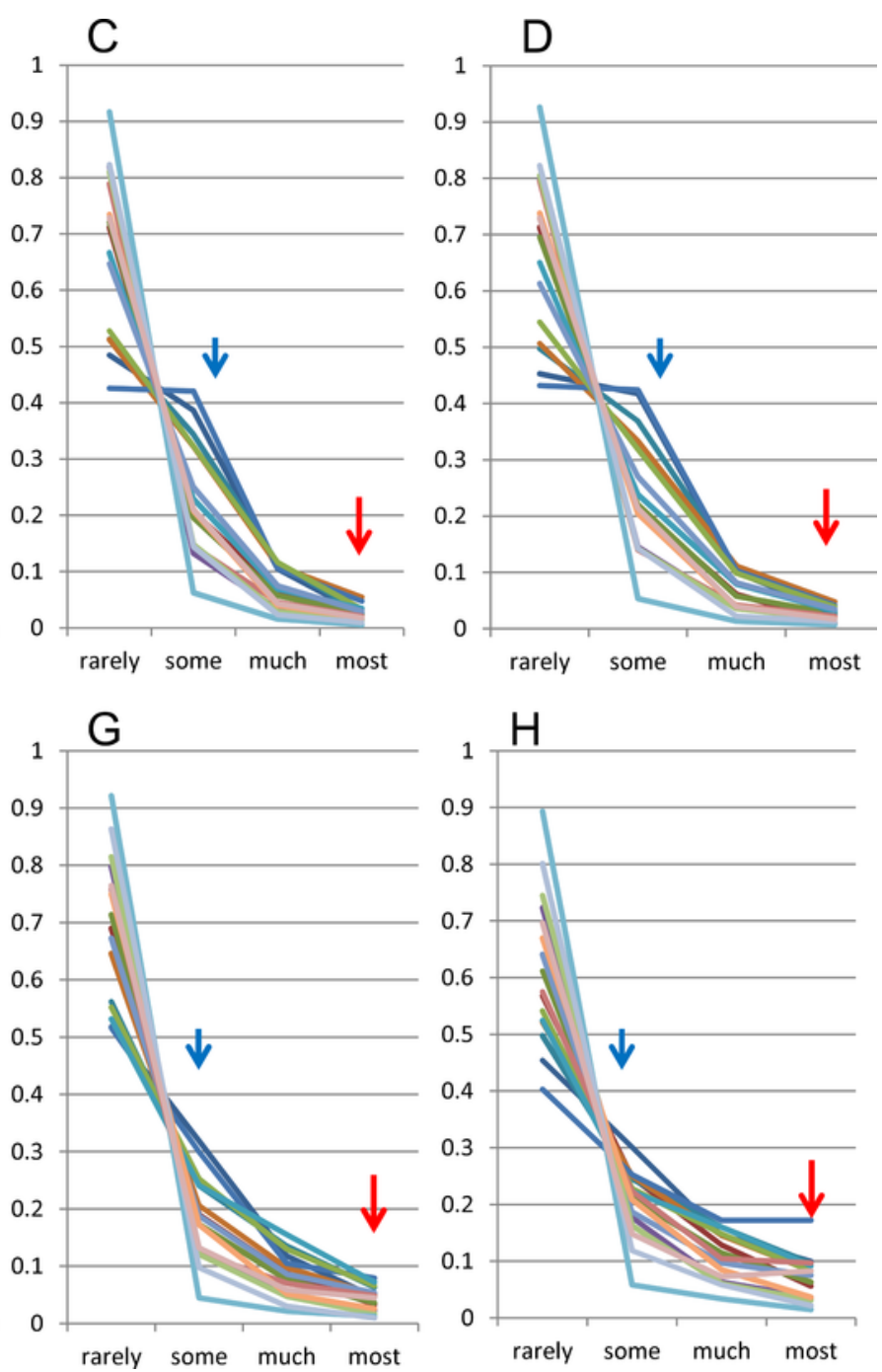
3

The distributions of the 16 items for "some" to "most" responses by age group using a log-normal scale.

The distributions of the 16 items for "some" to "most" responses by age group, A) 12-19, B) 20-29, C) 30-39, D) 40-49, E) 50-59, F) 60-69, G) 70-79, and H) 80-89 years. While the distribution of the 16 negative symptom items for "some" to "most" responses showed a parallel linear pattern across all age groups, the slopes of the lines for 16 negative items with a log-normal scale were seen to change according to age.
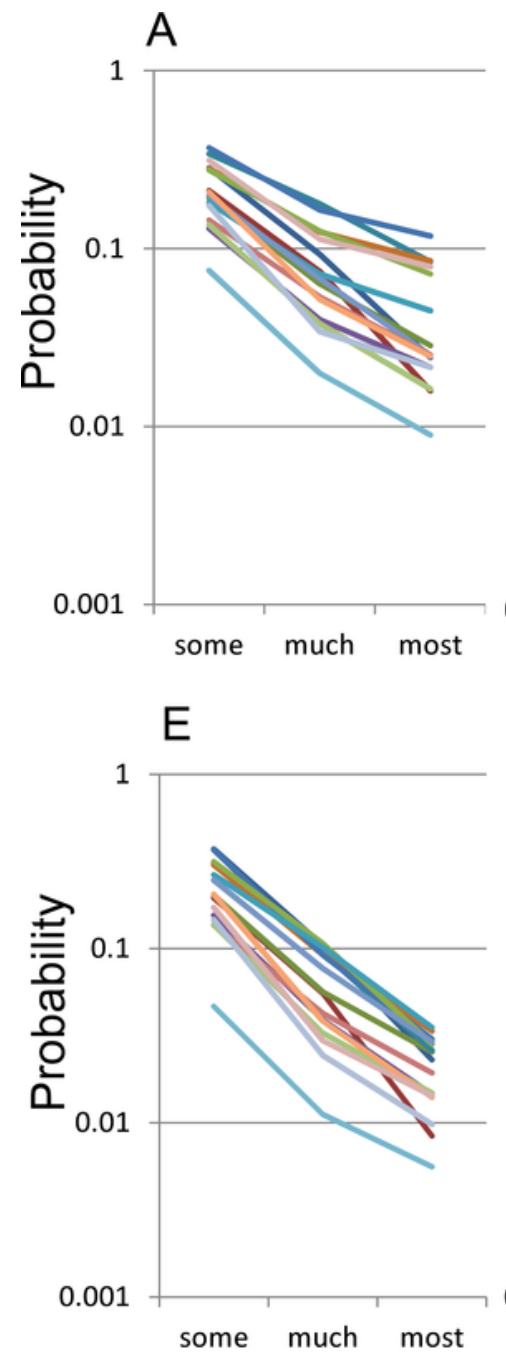

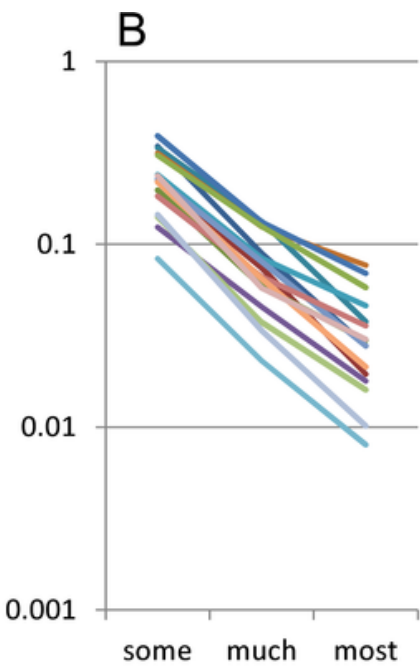

$\mathrm{F}$

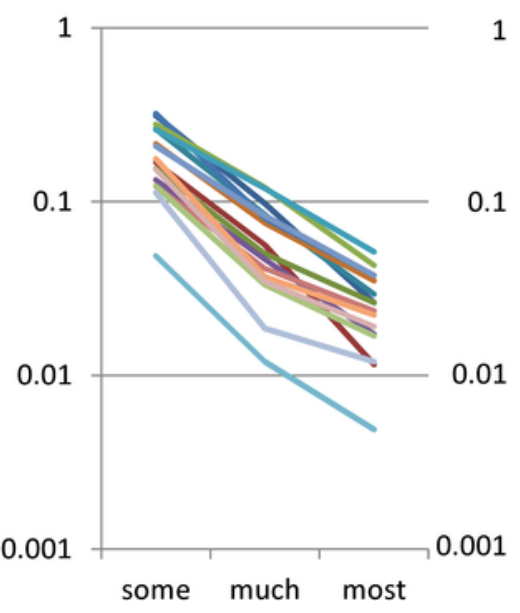

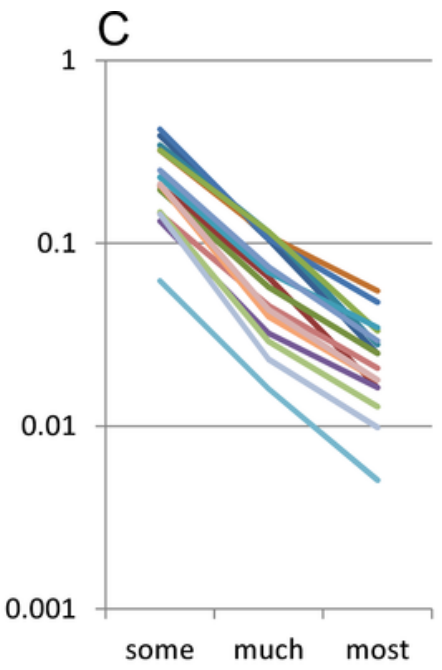

G

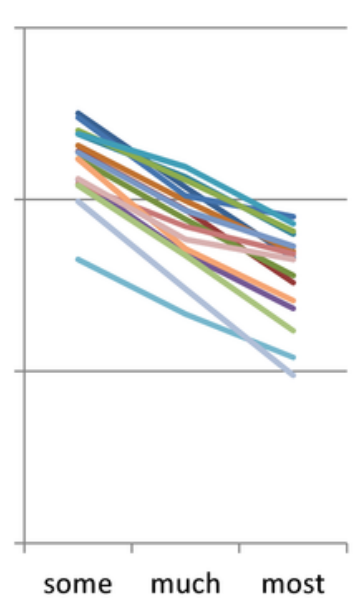

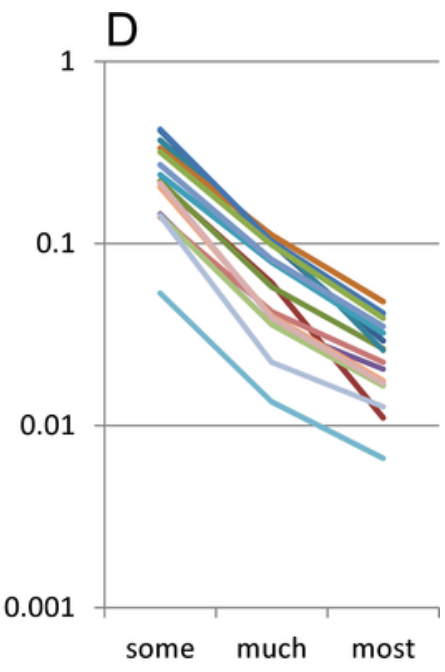

$\mathrm{H}$

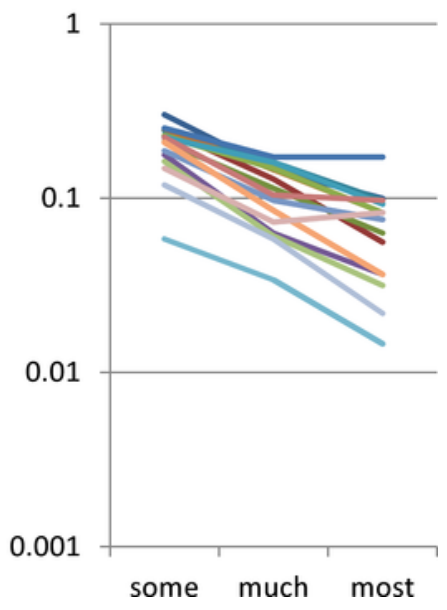




\section{4}

The relationships between age and estimated parameters $P$ and $r$.

Red graph indicate the the average of the estimated parameter $r$. Blue graph indicate the probability of "some." The average of the estimated parameter r exhibited a U-shaped pattern, being high during 12-29 years, staying low during 30-59 years, and then increasing again during 60-89 years. In contrast, the average of parameter $P$ slightly increased during 12-39 years, decreased during 40-79 years, and then slightly increased again during 80-89 years. 


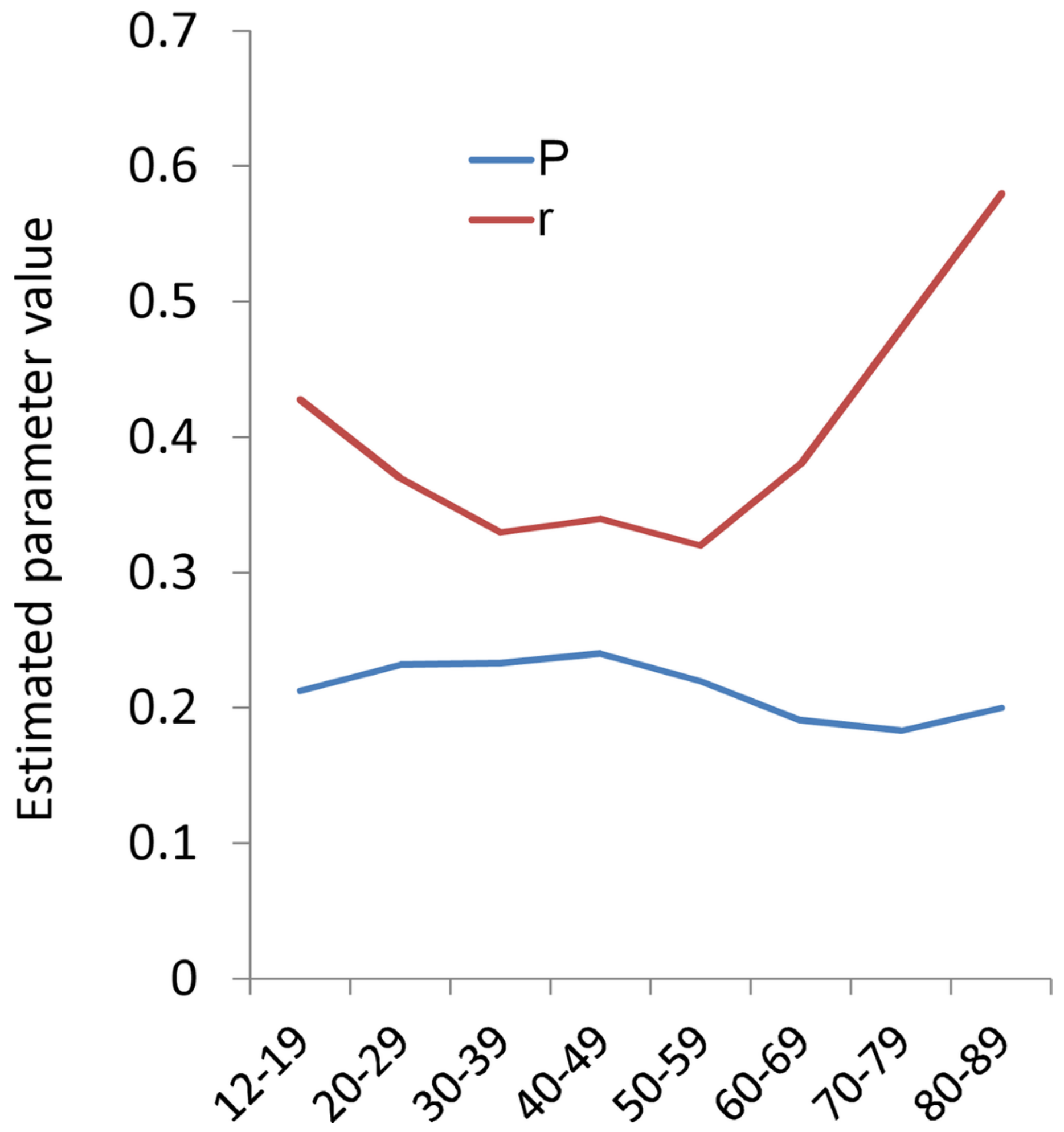




\section{5}

The relationships between age and the total scores of 20 items and 16 negative items using the Likert and binary methods.

A) In the standard Likert method, the total CES-D score and total 16-item score showed a Ushaped pattern. B) In the binary method, the total CES-D score and total 16-item score showed a downward trajectory with age.

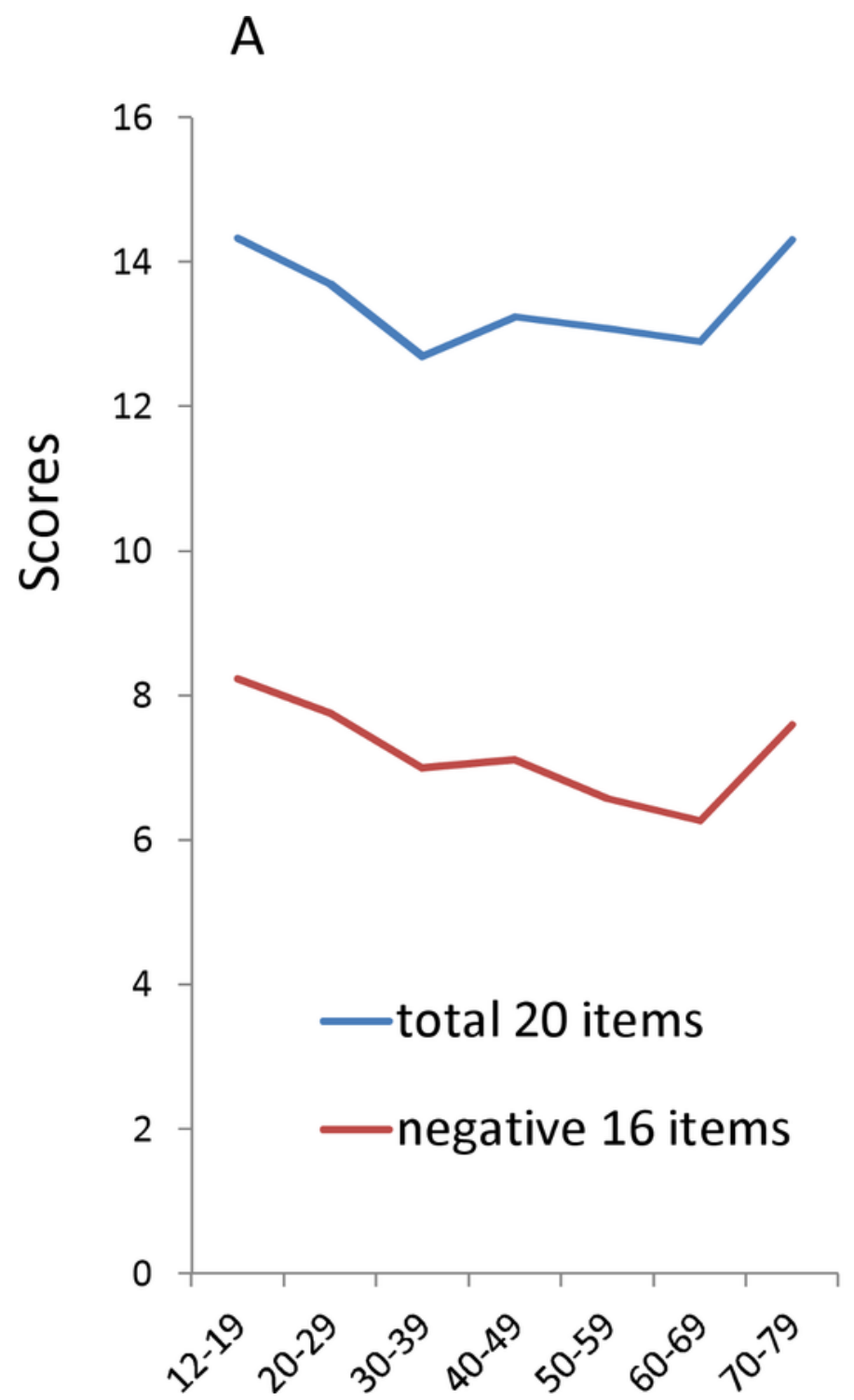

B

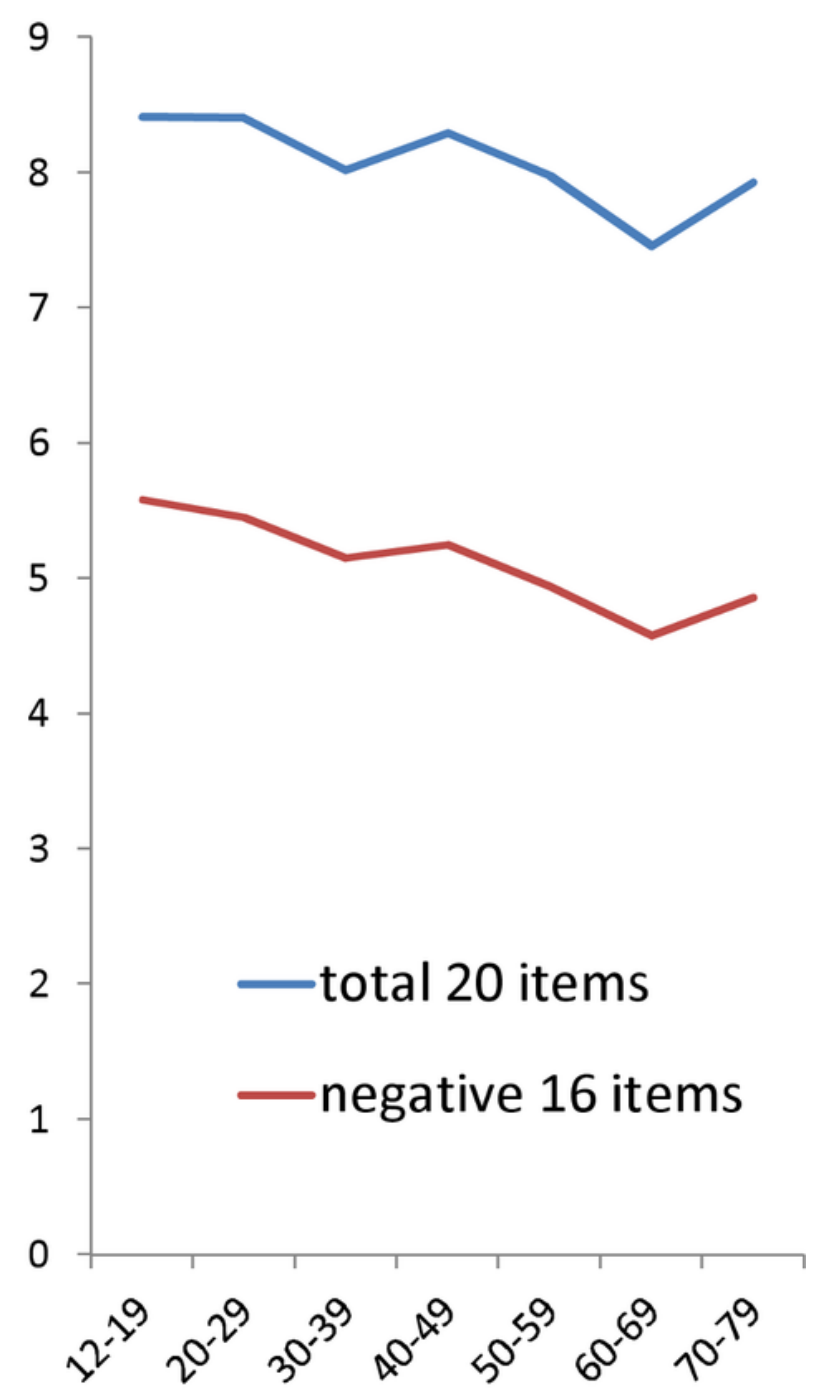




\section{Table 1 (on next page)}

Mean of each item according to age group in the general Japanese population.

* The lowest value. **The highest value. The positive symptom items are reverse-scored. 


\begin{tabular}{|c|c|c|c|c|c|c|c|c|c|}
\hline Number & Age group & $12-19$ & $20-29$ & 30-39 & $40-49$ & 50-59 & $60-60$ & 70-79 & 80-89 \\
\hline & \multicolumn{9}{|l|}{ Depressed mood } \\
\hline 3 & Blues & 0.41 & 0.40 & 0.39 & 0.42 & 0.39 & $* 0.33$ & 0.44 & $* * 0.63$ \\
\hline 6 & Depressed & 0.79 & 0.79 & 0.71 & 0.70 & 0.59 & $* 0.47$ & 0.55 & $* * 0.79$ \\
\hline 9 & Failure & 0.74 & 0.73 & 0.66 & 0.63 & $* 0.61$ & 0.65 & 0.71 & $* * 0.77$ \\
\hline 10 & Fearful & 0.27 & 0.27 & $* 0.24$ & 0.28 & 0.28 & 0.28 & 0.30 & $* * 0.41$ \\
\hline 14 & Lonely & 0.33 & 0.42 & 0.30 & 0.29 & 0.28 & $* 0.28$ & 0.41 & $* * 0.72$ \\
\hline 17 & Crying & 0.14 & 0.15 & 0.11 & 0.10 & $* 0.08$ & 0.09 & 0.12 & $* * 0.17$ \\
\hline \multirow[t]{2}{*}{18} & Sad & 0.39 & 0.41 & 0.34 & 0.34 & $* 0.32$ & 0.32 & 0.35 & $* * 0.49$ \\
\hline & \multicolumn{9}{|c|}{ Somatic symptoms and retarded activities } \\
\hline 1 & Bothered & $* 0.54$ & 0.61 & 0.67 & 0.71 & 0.65 & 0.59 & 0.69 & $* * 0.89$ \\
\hline 2 & Appetite & 0.41 & 0.43 & 0.39 & 0.37 & 0.33 & $* 0.32$ & 0.46 & $* * 0.67$ \\
\hline 5 & Trouble concentrating & 0.95 & 0.72 & 0.66 & 0.66 & 0.58 & $* 0.52$ & 0.70 & $* * 0.86$ \\
\hline 7 & Effort & 1.05 & 0.86 & 0.77 & 0.75 & 0.64 & $* 0.59$ & 0.75 & $* * 1.11$ \\
\hline 11 & Sleep & $* 0.46$ & 0.55 & 0.47 & 0.49 & 0.57 & 0.65 & 0.77 & $* * 0.82$ \\
\hline 13 & Talked & $* 0.41$ & 0.47 & 0.49 & 0.54 & 0.49 & 0.49 & 0.52 & $* * 0.60$ \\
\hline \multirow[t]{2}{*}{20} & Get going & $* * 0.78$ & 0.44 & 0.35 & 0.34 & $* 0.27$ & 0.28 & 0.38 & 0.54 \\
\hline & \multicolumn{9}{|l|}{ Interpersonal relations } \\
\hline 15 & Unfriendly & 0.26 & 0.26 & 0.24 & 0.26 & $* 0.25$ & 0.24 & 0.27 & $* * 0.38$ \\
\hline \multirow[t]{2}{*}{19} & Dislike & $* * 0.31$ & 0.24 & 0.22 & 0.23 & 0.22 & $* 0.18$ & 0.19 & 0.30 \\
\hline & \multicolumn{9}{|l|}{ Positive Affects } \\
\hline 4 & Good & 1.58 & 1.46 & *1.42 & 1.46 & 1.67 & $* * 1.74$ & 1.70 & 1.72 \\
\hline 8 & Hopeful & 1.74 & 1.52 & $* 1.47$ & 1.60 & 1.67 & 1.68 & 1.75 & $* * 1.87$ \\
\hline 12 & Happy & 1.63 & 1.64 & $* 1.53$ & 1.63 & 1.72 & 1.75 & $* * 1.76$ & 1.71 \\
\hline 16 & Enjoyed & *1.15 & 1.31 & 1.28 & 1.43 & 1.45 & 1.46 & 1.50 & $* * 1.54$ \\
\hline \multicolumn{2}{|c|}{ Total 16 negative items } & 8.2 & 7.8 & 7.0 & 7.1 & 6.6 & $* 6.3$ & 7.6 & $* * 10.2$ \\
\hline \multicolumn{2}{|c|}{ Total 4 positive items } & 6.1 & 5.9 & $* 5.7$ & 6.1 & 6.5 & 6.6 & 6.7 & $* * 6.8$ \\
\hline \multicolumn{2}{|c|}{ Total 20 items } & 14.3 & 13.7 & 12.7 & 13.2 & 13.1 & *12.9 & 14.3 & $* * 17.0$ \\
\hline
\end{tabular}

2 